\title{
The PRECISE (PREgnancy Care Integrating translational Science, Everywhere) database: open-access data collection in maternal and newborn health
}

\author{
Laura A. Magee ${ }^{*}$, Amber Strang ${ }^{1}$, Larry Li ${ }^{2}$ Domena Tu², Warancha Tumtaweetikul ${ }^{2}$, Rachel Craik1, \\ Marina Daniele ${ }^{1}$, Angela Koech Etyang ${ }^{3}$, Umberto D'Alessandro ${ }^{4}$, Ofordile Ogochukwu', Anna Roca ${ }^{4}$, \\ Esperança Sevene ${ }^{5,6}$, Paulo Chin ${ }^{6}$, Corssino Tchavana ${ }^{6}$, Marleen Temmerman ${ }^{3}$, Peter von Dadelszen ${ }^{1}$ and The \\ PRECISE Network
}

\begin{abstract}
In less-resourced settings, adverse pregnancy outcome rates are unacceptably high. To effect improvement, we need accurate epidemiological data about rates of death and morbidity, as well as social determinants of health and processes of care, and from each country (or region) to contextualise strategies. The PRECISE database is a unique core infrastructure of a generic, unified data collection platform. It is built on previous work in data harmonisation, outcome and data field standardisation, open-access software (District Health Information System 2 and the Baobab Laboratory Information Management System), and clinical research networks. The database contains globally-recommended indicators included in Health Management Information System recording and reporting forms. It comprises key outcomes (maternal and perinatal death), life-saving interventions (Human Immunodeficiency Virus testing, blood pressure measurement, iron therapy, uterotonic use after delivery, postpartum maternal assessment within $48 \mathrm{~h}$ of birth, and newborn resuscitation, immediate skin-to-skin contact, and immediate drying), and an additional 17 core administrative variables for the mother and babies. In addition, the database has a suite of additional modules for 'deep phenotyping' based on established tools. These include social determinants of health (including socioeconomic status, nutrition and the environment), maternal comorbidities, mental health, violence against women and health systems. The database has the potential to enable future high-quality epidemiological research integrated with clinical care and discovery bioscience.
\end{abstract}

Keywords: Open-source, Pregnancy, DHIS2, Placental disorders, eRegistry

\section{Background}

In less-resourced settings and particularly in subSaharan Africa, adverse pregnancy outcome rates are unacceptably high. This is reflected in global estimates of maternal mortality of 216/100,000 live births (2016 estimates), stillbirths of $18.4 / 1000$ live births (2015 estimates), and neonatal mortality of 18.0/1000 live births (2017 estimates) [1-3]. While estimates of near-miss

\footnotetext{
* Correspondence: Laura.A.Magee@kcl.ac.uk

'Department of Women and Children's Health, School of Life Course Sciences, Faculty of Life Sciences and Medicine, King's College London, Becket House, Room BH.05.11, 1 Lambeth Palace Road, London SE1 7EU, UK Full list of author information is available at the end of the article
}

morbidity for mothers and newborns are thought to be eight-fold higher $[4,5]$, there is no widespread routine collection of relevant outcome data as there is for mortality that allows for more accurate estimates globally, by region, and over time. Also, there is little data on health care quality and access to procedures that have the potential to improve these outcomes.

To effect improvements in maternal, newborn, and child health $(\mathrm{MNCH})$ outcomes, we need accurate epidemiological data about rates of death and morbidity, as well as the social determinants of health and processes of care. These data are needed from each country (and ideally 
region) to contextualise strategies. This is particularly important as countries are in different stages of the obstetric transition, with different health care infrastructures and priority needs [6]. Obtaining these data is currently hampered by numerous practical barriers, including a lack of standardisation of the predictor and outcome variables collected and their definitions [7]. There is keen interest in open-source software platforms, [8] self-programming and database revision.

The PRECISE (PREgnancy Care Integrating translational Science, Everywhere) Network is funded to collect epidemiological data and biological samples from women and babies in western (The Gambia), eastern (Kenya), and southern (Mozambique) sub-Saharan African countries. The aim is to understand, in the African setting, the predictors of placental disorders (hypertension, fetal growth restriction, and stillbirth) and determinants of their prognosis. The project will 'deep phenotype' around 10,000 pregnant women (and their babies), approximately 1500 of whom we anticipate will experience a pregnancy complicated by one of the placental disorders of interest (as above). (We will also collect data on around 1800 nonpregnant women of reproductive age as a control group.)

We have designed the PRECISE database with the following needs of the global research community in mind: (i) the movement away from purpose-built project databases so as to contain costs (initial and maintenance) and facilitate data-sharing [9]; (ii) the need for strategic support for large-scale, high-quality, epidemiological research; (iii) the desire to capitalise on the ubiquitous use of mobile technology for data collection in facility and in community; and (iv) the desire for open-access resources. We have built on previous work in outcome standardisation (by the International Consortium for Health Outcomes Measurement, ICHOM); data harmonisation (by the Global Pregnancy Collaboration, CoLab); the Manhiça Health and Demographics Surveillance System (HDSS), and an open-source, web-based health management information system (HMIS) platform (by the Norwegian Institute of Public Health).

In this manuscript, we describe our data collection platform of core and supplementary modules, with the potential to be used for high-quality epidemiological research. It is a single electronic repository of information (i.e. eRegistry) that can be shared by health system administrators, clinicians, clinical investigators, and discovery scientists through links with a laboratory information system.

\section{Methods}

Given the focus of the PRECISE Network on placental disorders, the starting point was the CoLab 's COLLECT database, designed for global studies in pre-eclampsia [10]. COLLECT's minimal and comprehensive data sets were reviewed for their appropriateness for data collection in our study sites, in terms of fields, definitions, and comprehensiveness for the purposes of the deep-phenotyping to be undertaken in PRECISE [11]. Also, we reviewed the software platform and connectivity requirements as we knew internet access to be intermittent.

In anticipation of the need for additional content and to enhance future usability, globally-recommended indicators in health management information systems for $\mathrm{MNCH}$ were identified and included, as component parts of key definitions (such as gestational age and blood pressure [BP] values), rather than reportable indicators that may require interpretation or judgement (such as a diagnosis of gestational hypertension) [12]. Variables were supplemented by review of the existing standardised data collection forms in each study site, for antenatal care (ANC), delivery, and ward care (antenatal or postnatal).

The content of supplementary modules was based on the holistic data needs of PRECISE (Fig. 1). These included the social determinants of health (e.g., socioeconomic status,

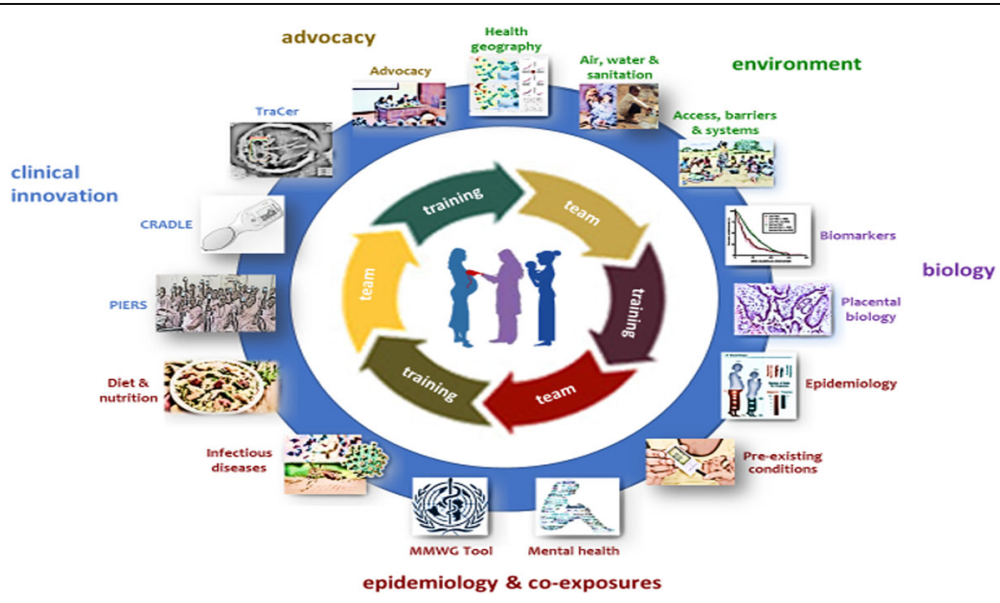

Fig. 1 PRECISE holistic approach to pregnancy research 
air quality, geography, or nutrition); maternal pre-existing and infectious co-morbidities; maternal mental health; and elements of health systems' strength (related to access, barriers, and quality of care). Standardised tools were identified whenever possible to address each of these data collection needs, based on needs-driven literature review (e.g., for maternal morbidity) and the experience of the PRECISE Network investigators.

The collection of biological specimens is recorded on an affiliated laboratory information management system (LIMS) described elsewhere. (For details of Baobab LIMS, please see Craik et al. Reprod Health 2019, in press [13].)

\section{Results}

The PRECISE protocol specifies prospective data collection into the PRECISE database, as pregnant women are enrolled at facilities for: (i) routine ANC (i.e., $\approx 10,000$ unselected pregnancies); or (ii) care of a placental disorder that is the focus of PRECISE (i.e., pregnancy hypertension, suspected fetal growth restriction [FGR], or intrauterine fetal death/stillbirth) if not already enrolled in PRECISE at that facility (i.e., $\approx 1500$ women). (Additional control women of reproductive age will be enrolled when they present for contraceptive counselling or in the company of another person who is presenting for ANC or other care (i.e., $\approx 1800$ women of reproductive age); in the Gambia, women of reproductive age will be recruited in the community.) [(For details of PRECISE Protocol, please see von Dadelszen et al. Reprod Health 2019, in press [14].) A project-specific eligibility screening has been designed to guide the study staff through the details of participant selection and data collection, documenting all information that would normally be recorded on a paper screening log, and eliminating the need for the study staff to carry paper guidance.

Depending on the site, not all staff have clinical training. However, all have been trained on the content and structure of the database, housed on android tablets (as described below). The sequence of data collection follows the flow of women through the clinic care system as much as possible.

\section{Data fields}

In choosing the content of the PRECISE database, we reviewed all variables in the COLLECT 'minimal' or core data set and the 'comprehensive' version of the database. We supplemented our review with other $\mathrm{MNCH}$ instruments for measurement of demographics, pre-pregnancy health or obstetric history, processes of care, or outcomes.

The 923 variables in PRECISE were organised according into the following modules: (i) general visit information, including identifiers and geoinformatics variables of relevance; (ii) baseline demographics and social determinants of health; (iii) past medical and obstetric histories; (iv) nutrition, an additional social determinant of health; (v) current pregnancy details, including addiction and maternal mental health; (vi) details of assessments of pregnancy hypertension, FGR, and intrauterine fetal death (IUFD)/ stillbirth; (vii) labour and delivery outcomes; (viii) maternal and newborn outcomes; and (ix) postpartum health and health care (Table 1). (Further details about the origins of each variable in each module are contained in Additional file 1, Tables S2A to S2N.)

The modules have the flexibility to be be grouped according to the data collection processes for PRECISE: screening, PRECISE visit 1 , PRECISE visit 2, information from other ANC visits, pregnancy outcomes (birth, maternal, and newborn), and details of presentation with placental disease (as relevant) or with laboratory results (as applicable at any visit), as shown in Table 2.

The 25 core components are focussed on intrapartum and immediate postpartum care as the time when most adverse outcomes cluster. The components would be appropriate for future use as screening data collection and would impose the lowest burden on the health system. These cover globally-recommended indicators in national HMIS recording and reporting forms, as per a review of data elements related to maternal and newborn health that were captured at different levels of the health system by HMIS tools from 14 USAID priority countries [12]. These core components consist of: (i) key mortality outcomes, of maternal death, stillbirth, and neonatal death (early and late); and (ii) life-saving interventions, of Human Immunodeficiency Virus (HIV) testing, BP measurement, iron therapy, uterotonic use after delivery, postpartum maternal assessment within $48 \mathrm{~h}$ of birth, and newborn resuscitation, immediate skin-to-skin contact, and immediate drying. Additional variables complete the 17 core administrative variables for the mother and baby. For the mother, these are: maternal age, parity, weight, substance use, need for intensive care as reflected by maternal near-miss morbidity, transfusion of blood products, and maternal length of stay. For the baby (ies), the core administrative variables are: spontaneous or iatrogenic preterm birth, sex, birthweight, birth injury, and obvious major birth defects and broad type), as well as location and mode of delivery [44]. These outcomes were developed by the ICHOM. To date, we have not included patient-reported health status, satisfaction with care, health care responsiveness, and birth experience, all of which would require specific data collection tools and be hampered by low health literacy at our study sites.

Given the deep-phenotyping mandate of PRECISE, these core components were supplemented by additional variables suitable for the immediate purpose of deepphenotyping in PRECISE, as well as for future use in 
Table 1 Content of modules

\begin{tabular}{|c|c|}
\hline Modules & Description and tools \\
\hline General visit information) & $\begin{array}{l}\text { Identifiers for future contact }{ }^{a} \text {, including Health Demographic Surveillance System (HDSS) numbers, as } \\
\text { relevant } \\
\text { Geo-informatic variables of relevance, including from where the woman travelled to seek care, and how that } \\
\text { travel was achieved (as performed in the Community-Level Interventions in Pre-eclampsia [CLIP] Mozambique } \\
\text { Trial) }[15,16]\end{array}$ \\
\hline $\begin{array}{l}\text { Baseline information \& social } \\
\text { determinants of health }\end{array}$ & $\begin{array}{l}\text { Maternal demographics and pre-existing morbidities (Demographic Health Survey } 7 \text { [17], COLLECT database } \\
\text { [11], and CLIP trials [18], informed by regionally-appropriate tribal categories in The Gambia, Kenya, and } \\
\text { Mozambique } \\
\text { Grameen Poverty Probability Index for Senegal (as a surrogate for The Gambia for which there is none), } \\
\text { Kenya, and Mozambique [19] } \\
\text { UN International Fund for Agricultural Development Multidimensional Poverty Assessment Tool, MPAT [20] } \\
\text { Education and occupation (as in the CLIP trials [18]) } \\
\text { Social supports and transport (as in the CLIP trials [18]) } \\
\text { Joint Monitoring Programme on Water Supply and Sanitation (JMP), WHO and UNICEF Multiple Indicator } \\
\text { Cluster Surveys (MICS)- Household Questionnaire } 2017 \text { [21] }\end{array}$ \\
\hline Past medical and obstetric histories & $\begin{array}{l}\text { COLLECT [11] } \\
\text { CLIP trials [18] } \\
\text { Demographic Health Survey } 7[17]\end{array}$ \\
\hline Nutrition & Dietary Diversity Score from the Healthy Life Trajectories Initiative (HeLTI) [22] \\
\hline Current pregnancy details & $\begin{array}{l}\text { COLLECT [11] } \\
\text { CLIP trials [18] } \\
\text { WHO recommendations on antenatal care for a positive pregnancy experience, } 2016 \text { [23] } \\
\text { Maternal Death Notification Form - South Africa 2014 [24] } \\
\text { Maternal mental health (WHO Maternal Woice Tool - antenatal care [25]) }\end{array}$ \\
\hline Details of placental disorders & $\begin{array}{l}\text { COLLECT [11] } \\
\text { CLIP trials [18] } \\
\text { fullPIERS [26] and miniPIERS }[27,28] \text { predictive models of adverse maternal and perinatal outcome in pre- } \\
\text { eclampsia } \\
\text { Details of stillbirth in INDEPTH standardised verbal autopsy tool }[29]\end{array}$ \\
\hline Labour and delivery & \multirow[b]{2}{*}{$\begin{array}{l}\text { COLLECT [11] } \\
\text { CLIP trials [18] } \\
\text { Adverse maternal outcome by Delphi consensus (fullPIERS [26] and iHOPE [30]) } \\
\text { World Health Organization. WHO Recommendations on Antenatal Care for a Positive Pregnancy Experience. } \\
\text { Geneva, Switzerland: World Health Organization, } 2016 \text { [23] } \\
\text { Preterm birth phenotype [31] } \\
\text { Intergrowth-21 standards for weight at birth [32] } \\
\text { Averting Maternal Death and Disability (AMDD) Needs Assessment Toolkit: Modules } 9 \text { (Chart review for } \\
\text { women with obstetric complications) and } 10 \text { (Chart review of newborn mortality) [33] } \\
\text { ICD-MM [International Classification of Disease-Maternal Mortality (ICD-MM [34]) } \\
\text { Maternal Death Notification Form - South Africa 2014 [24], Kenya 2017 [35] } \\
\text { WHO } 2011 \text { Maternal Near-Miss Morbidity Approach [4] informed by an African Delphi Consensus process [36] } \\
\text { WHO verbal autopsy tool for stillbirths [37], INDEPTH Standardized Verbal Autopsy questionnaire [29] } \\
\text { International Classification of Disease-Perinatal Mortality (ICD-PM [38] } \\
\text { Ministry of Health Perinatal Death Review Form - Kenya (2017) } \\
\text { WHO Making Every Baby Count initiative [39] }\end{array}$} \\
\hline Maternal and newborn outcomes & \\
\hline Postpartum health & $\begin{array}{l}\text { Post-traumatic Stress Disorder Checklist-Civilian Version, PCL-C [40] } \\
\text { WHO Maternal Woice tool - postnatal care [41] (for mental health, violence against women and other } \\
\text { maternal morbidity) } \\
\text { WHODAS tool 2.0 [42] (health functioning) } \\
\text { EN-SMILING tool [43] (early childhood development and infant nutrition) } \\
\text { DHS-7 (also infant nutrition) [17] }\end{array}$ \\
\hline
\end{tabular}

${ }^{\mathrm{a}}$ To be stripped by encryption when data are transmitted to the central servers at UBC

different locations (depending on needs and interest) or at different times (to monitor trends, depending on regional/national/global needs). The variables were defined according to various context-specific sources. COLLECT definitions were used whenever possible, for the 60/923 variables shared with the COLLECT minimal dataset; however, definitions were not always deemed feasible to use from COLLECT given response options that were not appropriate for our study settings (e.g., ethnicity categories that could not be mapped to context-specific options), unavailability of the intervention (e.g., invasive prenatal diagnosis), and/or the absence of robust clinical 
Table 2 Presentation of data collection in PRECISE database

\begin{tabular}{|c|c|c|}
\hline Icons in PRECISE (DHIS2) ${ }^{a}$ & Timing of data collection & Applicable modules \\
\hline & Screening (eligi-bility \& data collection (each visit) & (Study or setting-specific) \\
\hline & PRECISE visit 1 & $\begin{array}{l}\text { - General visit information } \\
\text { - Baseline demographics and social determinants of health } \\
\text { - Past medical and obstetric histories } \\
\text { - Nutrition } \\
\text { - Current pregnancy details }\end{array}$ \\
\hline & PRECISE visit 2 & $\begin{array}{l}\text { - General visit information } \\
\text { - Current pregnancy details }\end{array}$ \\
\hline & Other ANC visit (each) & - Current pregnancy details (basic) \\
\hline & Birth (labour and delivery, maternal and baby outcomes) & $\begin{array}{l}\text { - Labour and delivery } \\
\text { - Maternal and newborn outcomes }\end{array}$ \\
\hline & Presentation with placental disease (any visit) & - Details of placental disorders \\
\hline & Laboratory results (any visit) & (Relevant to all modules at any visit) \\
\hline
\end{tabular}

${ }^{a}$ The REDCap interface has a 'select event' list

records (e.g., family history of pre-eclampsia). A particular concern was the use in COLLECT of some overarching stem questions (such as occurrence of "maternal morbidity") that relied on high health literacy of the data collectors who only if answering 'yes', would see questions about specific morbidities; health literacy is variable among women, care-providers, and data collectors in our study settings. In contrast, we focussed on clinical data that care-providers routinely document, not "reportable indicators"; for example, care-providers record the BP measurement and use the system-generated date and gestational age to autogenerate a diagnosis of gestational hypertension at 20 weeks. This general principle was respected throughout, such as for maternal and neonatal near-miss morbidity $[4,5,45]$.

To ensure high-quality of data, programme rules were added to implement skip-logics and cross-validation rules for checking inconsistencies in real time. Each module has a 'See details' option, the programming of which is customised to provide the key details of relevance to the user.

The woman's study identifier is used to link her epidemiological data with biorepository samples tracked in Baobab LIMS, the LIMS chosen for the project given it is open-source and its development to facilitate harmonisation of biobanks across Africa, our geographical focus. In this way, a participant's personal health information is not attached to any specimen in the Baobab LIMS database. Executable, customised programmes have been created to be run periodically by the data manager to transmit biobanking core data into the database. (For details of Baobab LIMS, please see Craik et al. Reprod Health 2019, in press [13].) 


\section{Software}

The PRECISE database is based on the District Health Information System 2 (DHIS2) software and its application, Tracker. First, DHIS2 is widely used (by 67 low and middle-income countries) and, importantly, has been implemented in the Palestinian Territories as the national eRegistry for maternal and child health [46]. The Tracker application provides capacity for individual-level data entry. Second, Tracker is flexible, easily set-up, opensource, and accessed using a variety of tools, including android tablets and smartphones, to maximise flexibility of data collection at service delivery points [47]. If internet connectivity is lost, work can continue offline to facilitate efficient and effective data management. There is a 'share' function that generates a QR code (i.e., a machinereadable optical label that contains the relevant information about a PRECISE participant) that, if scanned by another device, will transfer the woman's information to another PRECISE tablet, allowing for data collection without interruption. Finally, the mandate of PRECISE includes capacity-building in Africa, and DHIS2 Tracker has the greatest potential to form the basis for an eRegistry. DHIS2 is packaged as a standard Java Web Archive file and runs on any Servlet container with Java Runtime Environment version 8 or higher installed, including the need for a web server and a database server with sufficient memory and storage space. As the preferred software environment for production server, Ubuntu 16.04 LTS operating system, PostgreSQL database and Tomcat Servlet are recommended. Training materials have been written so that those entering data have requisite skills to ensure accuracy [47].

Other software was considered. We found the structure of the COLLECT database challenging for the purposes of PRECISE. First, we had available to us either all of the field labels (variables and their characteristics) in the minimal dataset (i.e., $\approx 500$ ) or all in the comprehensive dataset (i.e., $\approx 5000$ ), but neither approach was realistic due to challenges with individual variables, as outlined above. Second, the MedSciNet software required continuous connectivity for data entry, but this is not available at our sites. We considered Open Data Kit, a mobile data management application that has been widely deployed in more than 130 countries [48]; however, the application (app) cannot handle frequent changes to the data model and we sought a resilient system for future use [49]. We considered Open Medical Record System, created as an open-source health software for low-resource settings [50]; while widely-used for HIV care, this is not the case for antenatal and postnatal care of mothers and babies. Finally, we considered Research Electronic Capture (REDCap) software, as it is widely used and open-access; however, REDCap does not have a personal dashboard, may not support well repeatable instruments and events, and the website has only a report tool with filters, rather than analytical tools needed for future functionality.

We did experience difficulties with the very large volume of questions to be asked in PRECISE which caused the DHIS2 Android Capture app to perform very slowly; a work-around was created to decrease the volume of data collection associated with any one 'icon' (as in Table 2), and this dramatically improved speed. However, in testing, the software was still not syncing $100 \%$ of all data entered, a problem experienced by other software users. In addition, the personal dashboard of a participant across multiple programmes (or tiles) was not always updated automatically if it was not opened in a programme. Given our need to start PRECISE data collection, we opted to begin on REDCap software that is compatible with the technical specifications of DHIS2 with which it has a similar look and feel (Fig. 2). Further testing will determine whether we revert to direct use of DHIS2, or remain with the REDCap interface.

In the PRECISE database, all participants are given a unique study identifier. Personal information is collected and held on a local database kept in a locked environment, with direct access restricted to authorised individuals who are responsible for monitoring and backing up the system. All other access to the database is controlled by logical security with access control and across a standard secure Hyper Text Transfer Protocol Secure network protected by a firewall and system controls with authentication, role-based authorisation, and audit trails. Strong password policies include password length and complexity restrictions. All database back-ups are encrypted and local Information Technology security policies and procedures followed.

Data collection tablets are password-protected and back-ups are encrypted. Each user logs into the app with a strong username and password, ensuring the study participant's confidentiality even with loss of tablets. Data are synchronised with local servers via secure connections, preferably daily. To ensure confidentiality, only authorised staff have access to personal information to facilitate data collection and cleaning. Loss or damage of a tablet that results in loss of data would be reported immediately. Information would be erased before any damaged tablets were recycled. Only password-protected computers running an antivirus program are used to download and process personal information for data linkage and cleaning.

All data are stored locally on high capacity servers stored in locked rooms. Extra measures for security include auxiliary power and continuous system monitoring. A tape back-up system is used for the database (and website). 
(a)

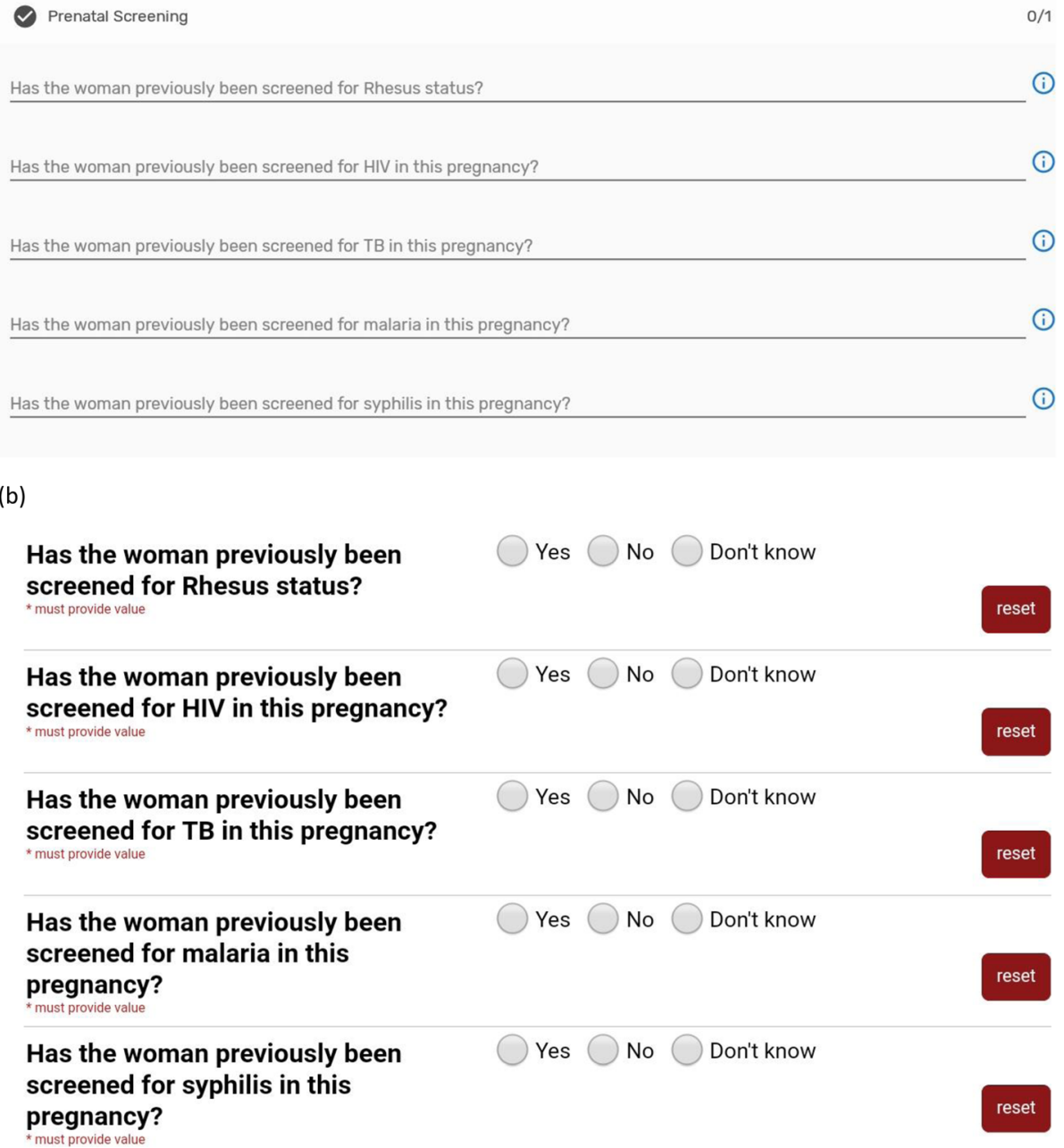

Fig. 2 Interfaces for (a) DHIS2 Tracker and (b) REDCap

A copy of all de-identified qualitative, clinical, geo-informatic, and laboratory data are stored on a remote server located at the British Columbia Children's Hospital Research Institute with daily backup and management by the PRE-EMPT team (PREgnancy Evidence, Monitoring, Partnerships and Treatment) at the University of British Columbia (UBC). Data are stored on a secure database on a high-speed network with access control, and servers are stored in a secure locked server room with restricted access to authorised system administrators. When data are sent for merging with master databases, research staff will keep a copy on their restricted network drive. The final dataset along with hard copies of results will be kept for at least 10 years.
For PRECISE, in each country, the database system is hosted and managed by local IT system administrators who are responsible for network security and access control, system updates, database back-up and recovery, and the in-country computer updates, in compliance with local IT network, system, and security policies. The data manager has set up and configured the database, tested the system functionalities, updated 'meta-data' (that help users find relevant information within the database), and installed/configured mobile apps on the tablets. The data manager is responsible for importing and exporting data, monitoring data updates, audit trails and reports, and resolving data queries within the study team.

It is the duty of sites to ensure data collected are complete and accurate and to run validation rules. Data 
are queried periodically by the local data manager to check for timely data collection and synchronisation, missing information, and discrepancies. Only deidentified data will be transmitted to the UBC central database, preferably on a weekly basis, through a secure connection with 256-bit Secure Sockets Layer encryption. At UBC, data queries are run and a query report with feedback provided to each site's local data managers. The data query report focusses on missing values without reasonable comments, out-of-range values, and data inconsistencies that violate validation rules. The report is designed to be reviewed and the queries resolved with the local team. Built-in audit trails provide historical records of data-update activities.

Compliance with General Data Protection Regulation (GDPR) is reliant on both the database and the operational policies, procedures and processes of the local study teams, to ensure that the necessary steps are taken to: obtain appropriate informed consent for data management (including collection, retention, sharing, disposal, and dispute); staff training; designation of a Data Protection Officer; tablet protection; server/platform protection; and detection and reporting of breaches. In addition, consent to participate in any project involving the database must protect participants' eight individual rights under GDPR to: be informed, access, rectification, withdrawal of consent, erasure, restriction of processing (of data), data portability, and objection.

\section{Challenges}

Our challenge related to our planned software platform, DHIS2, has been discussed above. Additional challenges relate to implementation of database content.

First, the World Health Organization (WHO) ANC guidelines recommend that, "Health-care providers should ask all pregnant women about their tobacco use (past and present) and exposure to second-hand smoke as early as possible in the pregnancy and at every antenatal care visit", and, "Health-care providers should ask all pregnant women about their use of alcohol and other substances (past and present) as early as possible in the pregnancy and at every antenatal care visit" [23]. Our in-country teams customised these questions to deal with cultural sensitivities.

Second, given the critical importance of mental health in pregnant and postpartum women, we had planned to integrate the WHO 'WOICE' tool for measurement of maternal morbidity, to which we added a scale of post-traumatic stress disorder [25, 41]. However, in-country teams were not comfortable due to the lack of both privacy in which to ask and answer these questions in the ANC setting, as well as the lack of well-established care pathways for women identified as having issues or being at high risk; antenatal and postnatal modules containing these questions are currently offline while we undertake care pathway mapping to prepare for the inclusion of these questions during the course of the project.

\section{Discussion}

The PRECISE database as designed is a unique infrastructure of a generic, unified data collection platform of core and supplementary variables that cover globally-recommended indicators included in HMIS recording and reporting forms, comprised of: key outcomes, life-saving interventions, and an additional 17 core administrative variables for the mother and babies. In addition, the database has a suite of additional variables designed for 'deep phenotyping' related to the placental disorders of hypertension, FGR, and stillbirth, but which are widely applicable for studying pregnancy-related conditions: social determinants of health, maternal co-morbidities, nutrition, mental health and domestic violence. The data elements and definitions have been standardised to comply with existing data dictionaries and/or tools, from international clinical, research, and policy organisations, including the $\mathrm{WHO}$, as well as efforts for international outcomes standardisation. The software platform is DHIS2, which is open-access and supported by free programming training by the University of Oslo as a WHO Collaborating Centre.

\section{Strengths and limitations}

Data collection at the point of clinical care is enabled by the modular structure of the PRECISE database. This approach to an electronic health record is both longitudinal and truly prospective, so collection of relevant clinical data will precede outcomes, which means that they cannot be biased by those outcomes. Specifically, predictors will be collected prior to occurrence of a placental disorder, and a placental disorder prior to the occurrence of maternal and perinatal mortality and morbidity. This is critical to understanding the epidemiology and true denominator and numerator of adverse outcomes, such as stillbirths, a major contributor to lives lost in Reproductive MNCH (RMNCH).

DHIS2, on which Tracker is programmed, is sustainable. The core software development team is hosted as a "global public good" at the University of Oslo, a world leader in HMIS strengthening in lessdeveloped countries; DHIS2 has been implemented, at least regionally, in 87 countries and Indian states, with 53 operating at national scale. The University of Oslo also contributes by offering in-country capacity 
building and implementation support and research, and as a WHO Collaborating Centre for Innovation and Implementation Research.

Limitations of the PRECISE database include how the questions must be structured; any responses for which 'mark all that apply' is appropriate must be phrased as different questions. Fields cannot currently be auto-filled from earlier data entries on the Android app; for example, if a woman has indicated that she is HIV positive on a previous form, Tracker will not automatically fill in later fields recording her HIV status. Also, images from supporting documentation (such as visual aids to describe sanitation facilities) cannot currently be uploaded and stored on the DHIS2 Android app. There is no offline data capture app that is supported on an Apple operating system device; however, the platform can still be accessible via internet through Safari or Chrome browsers if installed on an Apple device.

\section{The literature}

The WHO promotes client registry-based health information systems as one of the most promising avenues to support quality universal health coverage in $\mathrm{RMNCH}$. While most less-developed countries have national eHealth plans, a lack of harmonisation and capacity-building risks squandering opportunities for research and collaboration.

A standardised approach to $\mathrm{RMNCH}$ data field collection is critical. Measurement strategies must be consistent across communities and countries, and over time, in order to benchmark and address underlying differences, improve outcomes and reduce disparity. The Bill \& Melinda Gates Foundation (BMGF)-funded Healthy Birth, Growth, and Development - knowledge integration initiative is assembling 'big data' and novel analytic data rallies (focussed analytic efforts) from more than 170 BMGF-funded projects, involving $\approx 12$ million subjects from 34 countries, to inform key questions in child health and development [51]; the investigators will drill down through masses of data looking for patterns, statistical anomalies, and other newly discovered facts to inform new hypotheses in clinical research, and fashion updated clinical guidelines and protocols [52]. However, the initiative faces the challenge of different data fields, with different definitions, and different platforms - the same challenge faced by individual patient data meta-analyses in pregnancy. For example, in its synthesis of international angiogenic biomarker data (22 pregnancy cohorts, 16,462 pregnancies), the BMGF-funded CoLab estimates that more than $300 \mathrm{~h}$ were required to map data fields and definitions from one cohort to another, in order to find common ground on which to base the data synthesis [53].

Electronic health (eHealth) technology is the most frequently cited opportunity for maternal health among international researchers [54]. eHealth has been described as the ... use of information and communications technologies in support of health and health-related fields, including health care services, health surveillance, health literature, and health education, knowledge, and research [52]. At a global level, health data may: (i) not be captured at all; or if collected, (ii) captured but multiple times in multiple ways, or (iii) captured but in a way that cannot be shared due to interoperability issues or a lack of standardised definitions. This is not surprising given that approximately half of all referral facilities rely on paper data management [30]. The implications are illustrated by estimates of the maternal mortality ratio (per 100,000 live births) in Lesotho that vary by over 10-fold depending on the data source-100/100, 000 based on their confidential enquiry into maternal deaths, 500/100,000 according to entry of aggregate data from a paper birth registry into DHIS2, and 1057/100,000 as reported by the Demographic Health (household) Survey [Personal communication, B Pattinson 2018 Oct 4].

Making data valuable to the collector is the key to obtaining quality data, and lack of data use where it is collected has been cited as the weakest part of the HMIS in many countries [55]. In contrast, collecting data at point-of-care, in an electronic record with decision- and work-flow support, is designed with care providers in mind as both users and beneficiaries.

While many countries are implementing health registries in various forms, very few can act as an integrating backbone for health information and bioscience, clinical, and epidemiological research [56]. This is unrelated to the fact that the majority are in transition from paper-based data to digital data collection systems, but rather that even when fully implemented, the current bespoke 'top-down' solutions may not capture locallyrelevant determinants of outcomes or be inter-operable between countries. Additionally, local/regional 'bottomup' efforts or bespoke databases for specific research projects are not standardised and thus, unable to monitor national trends [52].

eRegistries are systems using information and communication technologies for the systematic longitudinal collection, storage, retrieval, analysis, and dissemination of uniform information on the health determinants and outcomes of individual persons, to serve diverse stakeholders, including those providing health care services or health surveillance, and those 
conducting research [56]. eRegistries are feasible and have been successfully implemented in $\mathrm{MNCH}$ [56]. Most undertake duplicate data collection on paper with transfer to an electronic database (e.g., Maternal Newborn Health Registry of the National Institutes of Health and BMGF-funded Global Health Network). However, some countries (e.g., Kenya) have published research based on primary data entry in fully electronic registries at national or regional levels, and individual records have also been linked with laboratory tests and biorepositories [56, 57], and a fully-functional eRegistry programmed on DHIS2 with decision support is currently being evaluated in the Palestinian Territories [46].

The strengths and operational challenges of DHIS2 have been recently reviewed systematically, in 20 studies from 11 countries [47]. The most important strengths were the technical features of the software (e.g., open-source); proper management of data (e.g., data entry at point of service provision); and application flexibility (e.g., ability to customise to meet local needs and sustainability of software); all are being leveraged in PRECISE. The most important operational challenges were management/leadership (e.g., proper planning for establishment) and training (e.g., of staff), sufficient workforce, appropriate communication infrastructure (e.g., internet), and the political, cultural, social and structural infrastructure (including linguistic challenges). All of these are being addressed in PRECISE, by staff hiring, training sessions and materials, close supervision, provision of enhanced internet capabilities (although not continuous), and a strongly supportive leadership, including local governmental officials.

While follow-up in PRECISE is currently to 6 weeks postpartum, it is critical to develop mechanisms to facilitate long-term follow-up. Conducting PRECISE in collaboration with the Manhiça Health Research Centre affords us the opportunity to link our database with the routine morbidity surveillance conducted for all children under 15 years of age who attend for inpatient or outpatient care in the area covered by their HDSS [58].

Finally, there is a need to understand which component(s) of packages of care are key elements of successful interventions. For example, the WHO eightcontact ANC model includes health promotion and nutritional interventions, and prevention and early detection of selected pregnancy-related conditions and concurrent diseases, including malaria, HIV, and tuberculosis. There are currently significant knowledge gaps that impede optimisation of care, given: the complexity of factors to consider; the need for countries to adapt recommendations based on their country context and populations' needs; and the inability of rigorous study methods (such as randomised trials) to evaluate each individual component of such packages, particularly in various settings. However, the volume and quality of data collected in an eRegistry can facilitate an understanding of the effects of multiple mediators, while accounting for unobserved confounders, using a counter-factual approach with multiple simulations from observed data, and bootstrapping for confidence intervals. Taking the eightvisit WHO ANC model, one could ask if it is necessary to measure proteinuria at every ANC visit? Or, what is the value of screening for hypertension at 32 weeks' gestation, in relation to other potential mediators of any observed effect (Fig. 2)?

\section{Conclusions}

We seek to build on PRECISE (in Africa) and the eRegistry concept by developing a generic, unified data collection platform that links clinical research and clinical care, thereby creating both a 'learning health system' for care-providers, and a monitoring, learning and evaluation system for academics and health administrators. Research could take the form of large-scale epidemiological work, clinical trials, implementation science, or discovery science by linking clinical data with stored biological samples logged in a LIMS system. Distributed analytical software packages (such as DataSHIELD) can now enable real-time, in-country analyses and research collaborations. In addition, there is potential to integrate decision support, facilitate remote access and analyses, and replace manual aggregation of data from multiple sources with electronically-generated reports for submission to central authorities, thus providing a mechanism for governments and other international agencies to monitor trends and identify priorities for more detailed data collection.

\section{Supplementary information}

Supplementary information accompanies this paper at https://doi.org/10. 1186/s12978-020-0873-8.

Additional file 1. Supplementary Database Tables S2A-S2N.

\section{Abbreviations}

ANC: Antenatal care; App: Application; BMGF: The Bill \& Melinda Gates Foundation; BP: Blood pressure; CoLab: Global Pregnancy Collaboration; DHIS2: District Health Information System 2; eHealth: Electronic health; eRegistry: Electronic registry; FGR: Fetal growth restriction; GDPR: General Data Protection Regulation; HDSS: Health and Demographics Surveillance System; HIV: Human Immunodeficiency Virus; HMIS: Health management information system; ICHOM: International Consortium for Health Outcomes Measurement; IUFD: Intrauterine fetal death; LIMS: Laboratory information management system; MNCH: Maternal, newborn, and child health; PRECISE: PREgnancy Care Integrating translational Science Everywhere, Network; PRE-EMPT: PREgnancy Evidence, Monitoring, Partnerships and Treatment; REDCap: Research Electronic Capture; RMNCH: Reproductive MNCH; UBC: University of British Columbia; WHO: World Health Organization 


\section{Acknowledgements}

We wish to thank the Global Pregnancy Collaboration for the opportunity to review the COLLECT database for use in PRECISE.

The PRECISE Network

\section{Members}

\section{In-country teams}

THE GAMBIA: Medical Research Council Unit The Gambia at the London School of Hygiene and Tropical Medicine, Fajara

KENYA: Aga Khan University, Nairobi

MOZAMBIQUE : Centro de Investigação em Saúde de Manhiça, Manhiça

\section{Central co-ordinating team}

Department of Women and Children's Health, School of Life Course Sciences, Faculty of Life Sciences and Medicine, King's

College London

Donna Russell Consulting

Co-Investigator team

Midlands State University, Zimbabwe

Kings College London

\section{University of Oxford}

London School of Hygiene and Tropical Medicine

St George's, University of London

University of British Columbia

University of Malawi
Umberto D'Alessandro, Anna Roca, Hawanatu Jah, Ofordile Oguchukwu, Andrew Prentice, Melisa MartinezAlvarez, Brahima Diallo, Adbul Sesey, Kodou Lette, Alpha Bah, Chilel Sanyang

Marleen Temmerman, Angela Koech Etyang, Peris Musitia, Mary Amondi, David Chege, Patricia Okiro, Geoffrey Omuse, Sikolia Wanyonyi

Esperança Sevene, Paulo Chin, Corssino Tchavana, Salesio Macuacua, Anifa Vala, Helena Boene, Lazaro Quimice, Sonia Maculuve, Eusebio Macete, Inacio Mandomando, Carla Carillho

Peter von Dadelszen, Laura A. Magee, Meriel Flint-O'Kane, Rachel Craik, Amber Strang, Marina Daniele

Donna Russell

Tatenda Makanga, Liberty Makacha, Yolisa Dube, Newton Nyapwere

Lucilla Poston, Jane Sandall, Rachel Tribe, Andrew Shennan, Sophie Moore, Tatiana Salisbury, Ben Barratt, Lucy Chappell, Sean Beevers, Kate Bramham

Hannah Blencowe, Veronique Filippi, Joy Lawn, Matt Silver, Matthew Chico

Judith Cartwright, Guy Whitley,

Marianne Vidler, Jing (Larry) Li, Jeff Bone, Mai-Lei (Maggie) Woo Kinshella, Beth A. Payne, Domena Tu, Warancha Tumtaweetikul

William Stones
Aris Papageorgiou, Alison Noble Sanjeev Krishna

\section{About this supplement}

This article has been published as part of Reproductive Health Volume 20 Supplement 1, 2020: Supplement special Issue on The PRECISE Network: Deep phenotyping of pregnancies in Africa. The full contents of the supplement are available online at https://reproductive-health-journal.biomedcentral.com/ articles/supplements/volume-20-supplement-1.

\section{Authors' contributions}

The database concept was conceived by LM and PVD. The manuscript was drafted by LM with editorial input from all named authors who reviewed and approved the final submitted version.

\section{Funding}

The project (including publication charges) was funded by a United Kingdom Research and Innovation GCRF GROW award (MR/P027938/1).

\section{Availability of data and materials}

The PRECISE Network Study are owned by the in-country teams. The PRECISE Network Data and Sample Access Committee, on which each in-country Principal Investigators (or designate) shall serve, shall provide guidance and oversight on the collection, processing and utilisation of specimens and data collected on behalf of the PRECISE Network Study.

\section{Ethics approval and consent to participate}

The PRECISE project was approved by the relevant research ethics committees for central co-ordinating activities at King's College London (KCL, HR-17/18-7855), and for in-country study activities at each site (2017-21), as follows: Farafenni, lliassa and Ngeyen Sanjal, co-ordinated by the Medical Research Council (MRC) Unit The Gambia (SCC 1619v1.2); Mariakani Sub-County Hospital and Rabai Health Centre, Kilifi County, co-ordinated by the Aga Khan University, Nairobi, Kenya (2018/REC-74(v2)); and Manhiça District Hospital and Xinavane Rural Hospital, Maputo Province, co-ordinated by Manhiça Health Research Centre (CISM), Mozambique (545/CNBS/18). Individual informed consent is provided by each participant for collection of her clinical data and relevant biological samples.

\section{Consent for publication}

All authors have reviewed and approved the manuscript for publication.

\section{Competing interests}

The PRECISE database was designed by the authors. The authors do not hold any financial interests (institutional or personal) related to the use of the PRECISE database. Completed disclosure of interest forms are available to view online as supporting documentation.

\section{Author details}

'Department of Women and Children's Health, School of Life Course Sciences, Faculty of Life Sciences and Medicine, King's College London, Becket House, Room BH.05.11, 1 Lambeth Palace Road, London SE1 7EU, UK. ${ }^{2}$ Department of Obstetrics \& Gynaecology, Faculty of Medicine, University of British Columbia, Vancouver, Canada. ${ }^{3}$ Centre of Excellence in Women \& Child Health, East Africa, Aga Khan University, Nairobi, Kenya. ${ }^{4}$ Medical Research Council Unit The Gambia at the London School of Hygiene and Tropical Medicine, Fajara, The Gambia. ${ }^{5}$ Department of Physiological Science, Clinical Pharmacology, Faculty of Medicine, Universidade Eduardo Mondlane, Maputo, Mozambique. ${ }^{6}$ Centro de Investigação em Saúde de Manhiça, Manhiça, Mozambique.

Published: 30 April 2020

\section{References}

1. Alkema L, Chou D, Hogan D, Zhang S, Moller AB, Gemmill A, et al. Global, regional, and national levels and trends in maternal mortality between 1990 and 2015, with scenario-based projections to 2030: a systematic analysis by the UN maternal mortality estimation inter-agency group. Lancet. 2016; 387(10017):462-74.

2. Blencowe H, Cousens S, Jassir FB, Say L, Chou D, Mathers C, et al. National, regional, and worldwide estimates of stillbirth rates in 2015, with trends from 2000: a systematic analysis. Lancet Glob Health. 2016;4(2):e98-e108.

3. World Health Organization. Global health observatory data. 2017. https:// www.who.int/gho/child_health/mortality/neonatal/en/.

4. Evaluating the quality of care for severe pregnancy complications - the WHO near-miss approach for maternal health; 2011. https://www.who.int/ reproductivehealth/publications/monitoring/9789241502221/en/. Accessed 20 Mar 2019.

5. Santos JP, Cecatti JG, Serruya SJ, Almeida PV, Duran P, Mucio B, et al. Neonatal near miss: the need for a standard definition and appropriate criteria and the rationale for a prospective surveillance system. Clinics (Sao Paulo). 2015;70(12):820-6.

6. Souza JP, Tuncalp O, Vogel JP, Bohren M, Widmer M, Oladapo OT, et al. Obstetric transition: the pathway towards ending preventable maternal deaths. BJOG. 2014;121(Suppl 1):1-4. 
7. Myers JE, Myatt L, Roberts JM, Redman C. COLLECT, a collaborative database for pregnancy and placental research studies worldwide. BJOG. 2019;126(1): 8-10.

8. REDCap (Research Electronic Data Capture). https://www.project-redcap. org/.2019.Mar.7. Accessed 20 Mar 2019.

9. Report by the Director General 71st World Assembly. mHealth. Use of appropriate digital technologies for public health. 2018.

10. Global Pregnancy CoLaboratory COLLECT database. https://pre-empt.bcchr. ca/collaboration/global-pregnancy-collaboration.2019.Mar.7. Accessed 20 Mar 2019.

11. Myatt L, Redman CW, Staff AC, Hansson S, Wilson ML, Laivuori H, et al, Strategy for standardization of preeclampsia research study design. Hypertension. 2014;63(6):1293-301.

12. Health Management Information Systems Review. Survey on data availability in electronic systems for maternal and newborn health in 24 USAID priority countries: Maternal and Child Survival Program; 2016. www. mcsprogram.org.

13. Craik $R$, et al. Baobab laboratory information management system. BMC Reprod Health. 2019; (submitted).

14. von Dadelszen P, Flint-O'Kane M, Poston L, Craik R, Russell D, Tribe RM, et al. The PRECISE (PREgnancy Care Integrating translational Science, Everywhere) Protocol. Reprod Health. 2019; (in press).

15. Makanga PT, Sacoor C, Schuurman N, Lee T, Vilanculo FC, Munguambe K, et al. Place-specific factors associated with adverse maternal and perinatal outcomes in southern Mozambique: a retrospective cohort study. BMJ Open. 2019;9(2):e024042.

16. Makanga PT, Schuurman N, Sacoor C, Boene HE, Vilanculo F, Vidler M, et al. Seasonal variation in geographical access to maternal health services in regions of southern Mozambique. Int J Health Geogr. 2017;16(1):1.

17. The DHS Program (Demographic and Health Surveys); 2018. https://www. dhsprogram.com/What-We-Do/Survey-Types/DHS-Questionnaires.cfm. Accessed 20 Mar 2019

18. The Community-Level Intervention in Pre-eclampsia (CLIP) Trials; 2019. https://pre-empt.bcchr.ca/treatment/clip-community-level-interventions-preeclampsia. Accessed 20 Mar 2019

19. Grameen Poverty Probability Index; 2018. https://www.povertyindex.org/. Accessed 20 Mar 2019.

20. The Multidimensional Poverty Assessment Tool (MPAT); 2014. https://www.ifad. org/en/web/knowledge/publication/asset/39631564. Accessed 20 Mar 2019.

21. UNICEF Multiple Indicator Cluster Surveys (MICS) - Household Questionnaire; 2019. https://washdata.org/unicef-multiple-indicator-cluster-surveys-mics. Accessed 20 Mar 2019.

22. The Health Life Trajectories Initiative (HeLTI); 2019. http://www.cihr-irsc.gc. ca/e/50275.html. Accessed 20 Mar 2019.

23. World Health Organization, Geneva; 2016. https://apps.who.int/iris/ bitstream/handle/10665/250796/9789241549912-eng.pdf;jsessionid=61A96E4 C86451A3213193FADACD213CA? sequence $=1$.

24. Maternal Death Notification Form - South Africa. https://www.who.int/ maternal_child_adolescent/epidemiology/maternal-death-surveillance/casestudies/south-africa/en/.2014. Accessed 20 Mar 2019

25. Maternal Woice Tool: Antenatal care; 2018. https://www.who.int/ reproductivehealth/MM_ANC_questionnaire.pdf?ua=1. Accessed 20 Mar 2019.

26. von Dadelszen P, Payne B, Li J, Ansermino JM, Broughton PF, Cote AM, et al. Prediction of adverse maternal outcomes in pre-eclampsia: development and validation of the fullPIERS model. Lancet. 2011;377(9761):219-27.

27. Payne BA, Hutcheon JA, Ansermino JM, Hall DR, Bhutta ZA, Bhutta SZ, et al. $A$ risk prediction model for the assessment and triage of women with hypertensive disorders of pregnancy in low-resourced settings: the miniPIERS (Pre-eclampsia Integrated Estimate of RiSk) multi-country prospective cohort study. PLoS Med. 2014;11(1):e1001589.

28. Payne BA, Groen H, Ukah UV, Ansermino JM, Bhutta Z, Grobman W, et al. Development and internal validation of a multivariable model to predict perinatal death in pregnancy hypertension. Pregnancy Hypertens. 2015;5(4): 315-21

29. INDEPTH Standardized Verbal Autopsy Questionnaire; 2003. http://www. indepth-network.org/resources/indepth-standardized-verbal-autopsyquestionnaire. Accessed 20 Mar 2019.

30. Duffy JM, van't Hooft J, Gale C, Brown M, Grobman W, Fitzpatrick R, et al. A protocol for developing, disseminating, and implementing a core outcome set for pre-eclampsia. Pregnancy Hypertens. 2016;6(4):274-8.
31. Villar J, Papageorghiou AT, Knight HE, Gravett MG, lams J, Waller SA, et al. The preterm birth syndrome: a prototype phenotypic classification. Am J Obstet Gynecol. 2012;206(2):119-23.

32. Intergrowth-21 standards for weight at birth; 2014. https://intergrowth21. tghn.org/newborn-size-birth/. Accessed 20 Mar 2019.

33. Averting Maternal Death and Disability (AMDD) needs assessment toolkit; 2015. https://www.mailman.columbia.edu/research/averting-maternal-deathand-disability-amdd/toolkit. Accessed 20 Mar 2019.

34. The WHO Application of ICD-10 to deaths during pregnancy, childbirth and the puerperium: ICD-MM; 2012. https://apps.who.int/iris/bitstream/handle/1 0665/70929/9789241548458_eng.pdf?sequence=1. Accessed 20 Mar 2019.

35. Republic of Kenya MoH. Maternal death review form (MDRF) MOH 372. 2017. www.health.go.ke

36. Tura AK, Stekelenburg J, Scherjon SA, Zwart J, van den Akker T, van Roosmalen J, et al. Adaptation of the WHO maternal near miss tool for use in sub-Saharan Africa: an international Delphi study. BMC Pregnancy Childbirth. 2017;17(1):445.

37. Aggarwal AK, Jain V, Kumar R. Validity of verbal autopsy for ascertaining the causes of stillbirth. Bull World Health Organ. 2011;89(1):31-40.

38. Lavin T, Allanson ER, Nedkoff L, Preen DB, Pattinson RC. Applying the international classification of diseases to perinatal mortality data, South Africa. Bull World Health Organ. 2018;96(12):806-16.

39. World Health Organization, Geneva; 2016. https://www.who.int/maternal child_adolescent/documents/stillbirth-neonatal-death-review/en/.

40. Post-traumatic stress disorder (PTSD) checklist - civilian version, PCL-C; 2003. https://www.mirecc.va.gov/docs/visn6/3_ptsd_checklist_and_scoring.pdf. Accessed 20 Mar 2019

41. Maternal Woice tool: Postnatal care; 2018. https://www.who.int/ reproductivehealth/MM_PPC_questionnaire.pdf. Accessed 20 Mar 2019.

42. WHO Disability Assessment Schedule 2.0 (WHODAS 2.0). World Health Organization, Geneva; 2010. https:/www.who.int/classifications/iff/whodasii/en/.

43. World Health Organization, Geneva; 2016. http://goldencommunity.org.np/ en-smile/

44. The International Consortium for Health Outcomes Measurement (ICHOM) standard set for pregnancy and childbirth; 2017. https://www.ichom.org/ portfolio/pregnancy-and-childbirth/. Accessed 20 Mar 2019.

45. Santos JP, Pileggi-Castro C, Camelo JS Jr, Silva AA, Duran P, Serruya SJ, et al. Neonatal near miss: a systematic review. BMC Pregnancy Childbirth. 2015:15:320.

46. Venkateswaran M, Morkrid K, Ghanem B, Abbas E, Abuward I, Baniode M, et al. eRegQual-an electronic health registry with interactive checklists and clinical decision support for improving quality of antenatal care: study protocol for a cluster randomized trial. Trials. 2018;19(1):54

47. Dehnavieh R, Haghdoost A, Khosravi A, Hoseinabadi F, Rahimi H, Poursheikhali A, et al. The District Health Information System (DHIS2): a literature review and meta-synthesis of its strengths and operational challenges based on the experiences of 11 countries. Health Inf Manag. 2018. https://doi.org/10.1177/1833358318777713.

48. Open Data Kit; 2019. https://opendatakit.org/. Accessed 20 Mar 2019.

49. Brunette W, Sudar S, Sundt M, Larson C, Beorse J, Anderson R. Open Data Kit 2.0: a services-based application framework for disconnected data management; 2017. https://doi.org/10.1145/3081333.3081365.

50. Open Medical Record System (MRS); 2019. https://openmrs.org/. Accessed 20 Mar 2019.

51. Healthy Birth, Growth \& Development (HBGDki); 2019. http://hbgdki.org. Accessed 20 Mar 2019

52. Haazen DS, et al. eHealth technical paper. M-A4Health (Measurement and accountability for results in health): a common agenda for the post-2015 era. 2015. https://www.who.int/healthsystems/news/MA4Health/en/.

53. Burke O, Benton S, Szafranski P, von Dadelszen P, Buhimschi SC, Cetin I, et al. Extending the scope of pooled analyses of individual patient biomarker data from heterogeneous laboratory platforms and cohorts using merging algorithms. Pregnancy Hypertens. 2016;6(1):53-9.

54. Kendall T. Critical maternal health knowledge gaps in low- and middleincome countries for post-2015: researchers' perspectives. Boston: Harvard T H Chan School of Public Health; 2015

55. Ndabarora E, Chipps JA, Uys L. Systematic review of health data quality management and best practices at community and district levels in LMIC. Inf Dev, 2013:30:103.

56. Froen JF, Myhre SL, Frost MJ, Chou D, Mehl G, Say L, et al. eRegistries: electronic registries for maternal and child health. BMC Pregnancy Childbirth. 2016;16:11. 
57. Bjerregaard-Andersen M, Gomes MA, Joaquim LC, Rodrigues A, Jensen DM, Christensen $\mathrm{K}$, et al. Establishing a twin registry in Guinea-Bissau. Twin Res Hum Genet. 2013;16(1):179-84.

58. Sacoor C, Nhacolo A, Nhalungo D, Aponte JJ, Bassat Q, Augusto O, et al. Profile: Manhica Health Research Centre (Manhica HDSS). Int J Epidemiol. 2013;42(5):1309-18.

\section{Publisher's Note}

Springer Nature remains neutral with regard to jurisdictional claims in published maps and institutional affiliations.

- fast, convenient online submission

- thorough peer review by experienced researchers in your field

- rapid publication on acceptance

- support for research data, including large and complex data types

- gold Open Access which fosters wider collaboration and increased citations

- maximum visibility for your research: over $100 \mathrm{M}$ website views per year

At BMC, research is always in progress. 